\title{
Ferromagnetism in a lattice of Bose condensates
}

\author{
Han $\mathrm{Pu}$, Weiping Zhang, and Pierre Meystre \\ Optical Sciences Center, The University of Arizona, Tucson, AZ 85721
}

(November 6, 2018)

\begin{abstract}
We show that an ensemble of spinor Bose-Einstein condensates confined in a one dimensional optical lattice can undergo a ferromagnetic phase transition and spontaneous magnetization arises due to the magnetic dipole-dipole interaction. This phenomenon is analogous to ferromagnetism in solid state physics, but occurs with bosons instead of fermions.

PACS numbers: 03.75.Fi, 75.45.+j, 75.60.Ej
\end{abstract}

The Heisenberg model of spin-spin interactions is considered as the starting point for understanding many complex magnetic structures in solids. In particular, it explains the existence of ferromagnetism and antiferromagnetism at temperatures below the Curie temperature. It is defined by the spin Hamiltonian [1]

$$
H_{\text {spin }}=-\sum J_{i j} \mathbf{S}_{i} \cdot \mathbf{S}_{j}
$$

where $\mathbf{S}_{i}$ is the spin operator for $i$-th electron, and $J_{i j}$ are known as the exchange coupling constants. This Hamiltonian arises from the direct Coulomb interaction among electrons and the Pauli exclusion principle. In addition to the exchange interaction, there exists another important type of magnetic interaction, the magnetic dipole-dipole interaction. However, in solid materials the dipolar coupling is typically several orders of magnitude weaker than the exchange coupling, and would correspond to Curie temperatures much below the observed ones. Hence its contribution to the spin Hamiltonian can be neglected in practice. It follows from this argument that ferromagnetism is not generally expected to occur in bosonic lattices of neutral atoms, a result of the inapplicability of the Pauli principle, the absence of Coulomb interaction and small atomic magnetic dipole moments. However, we qualify this remark by noting that as a result of accurate recent measurements of the scattering length of spin-changing collisions, it is now established that the ground state of optically trapped ${ }^{87} \mathrm{Rb}$ spinor Bose condensates is ferromagnetic, an important result that we use in the following [2,3].

The goal of this paper is to show that this result, combined with the recent experimental realization of regular arrays of Bose-Einstein condensates in optical lattices, leads to a situation where it becomes possible to carry out detailed static and dynamic studies of magnetism on one to three-dimensional periodic lattices.

Quantum degenerate Bose gases on optical lattices were first used to demonstrate "mode-locked" atom lasers

\footnotetext{
${ }^{1}$ We note however that the magnetic dipole-dipole interaction plays an important role in domain formation in macroscopic samples.
}

[4]. It has also been theoretically demonstrated that they undergo a Mott insulator phase transition as the depth of the lattice wells is increased [5]. Recently, they have become of interest in the study of quantum chaos [6,7]. Here, we show that spinor condensates, localized in optical lattices deep enough for the individual sites to be independent, can undergo a ferromagnetic-like phase transition that leads to a "macroscopic" magnetization of the condensate array.

We consider specifically the case of spinor ${ }^{87} \mathrm{Rb}$ condensates [8], which are as we have mentioned individual ferromagnets of random directions in the absence of external fields and magnetic dipole-dipole interaction. We show that the magnetic dipole-dipole interaction between lattice sites can spontaneously align the magnetization of the individual sites. This is possible because of the Bose enhanced magnetic dipole moments of the condensate which in turn enhances the strength of the magnetic dipolar interaction.

Our starting point is the Hamiltonian $H$ describing an $F=1$ spinor condensate at zero temperature trapped in an optical lattice, subject to a magnetic dipole-dipole interaction $H_{d d}$ and is coupled to an external magnetic field via the magnetic dipole Hamiltonian $H_{B}$ [9-12],

$$
H=H_{0}+H_{d d}+H_{B} .
$$

Here

$$
\begin{aligned}
H_{0} & =\int d^{3} r \psi_{\alpha}^{\dagger}(\mathbf{r})\left[-\hbar^{2} \nabla^{2} / 2 m+V_{L}(\mathbf{r})\right] \psi_{\alpha}(\mathbf{r}) \\
& +\left(\lambda_{s} / 2\right) \int d^{3} r \psi_{\alpha}^{\dagger}(\mathbf{r}) \psi_{\beta}^{\dagger}(\mathbf{r}) \psi_{\beta}(\mathbf{r}) \psi_{\alpha}(\mathbf{r}) \\
& +\left(\lambda_{a} / 2\right) \int d^{3} r \psi_{\alpha}^{\dagger}(\mathbf{r}) \psi_{\mu}^{\dagger}(\mathbf{r}) \mathbf{F}_{\alpha \beta} \cdot \mathbf{F}_{\mu \nu} \psi_{\nu}(\mathbf{r}) \psi_{\beta}(\mathbf{r})
\end{aligned}
$$

describes the interaction of the atoms with the lattice potential $V_{L}(\mathbf{r})$ and ground state collisions. It includes an implicit sum over the indices $\{\alpha, \beta, \mu, \nu\}=\{0, \pm 1\}$ that label the three Zeeman sublevels. The parameters $\lambda_{s}$ and $\lambda_{a}$ characterize the short-range spin-independent and spin-changing $s$-wave collisions, respectively. Specifically, $\lambda_{a}$ is proportional to the difference between the $s$-wave scattering lengths in the triplet and singlet channels 10 12]. It has recently been measured to be negative 
for ${ }^{87} \mathrm{Rb}$, accounting for its ferromagnetic ground state [2].

Our model includes the long-range magnetic dipoledipole interaction between different lattice sites, but neglect it within each site, assuming that it is much weaker than the $s$-wave interaction described by $H_{0}$. We also assume that the optical lattice potential is deep enough that there is no spatial overlap between the condensates at different lattice sites. We can then expand the atomic field operator as $\psi(\mathbf{r})=\sum_{i} \sum_{\alpha=0, \pm 1} \hat{a}_{\alpha}(i) \phi_{i}(\mathbf{r})$ where $i$ labels the lattice sites.

The Hartree wave function $\phi_{i}(\mathbf{r})$, determined by minimizing the total energy, is the wave function of the condensate at the $i$-th site. It is assumed that all Zeeman components share the same spatial wave function. If the condensates at each lattice sites contain the same number $N$ of atoms, then the ground-state wave functions for different sites have the same form $\phi_{i}(\mathbf{r})=\phi\left(\mathbf{r}-\mathbf{r}_{\mathbf{i}}\right)$. Under this condition, the dipolar interaction potential is $H_{d d}=\sum_{i, j \neq i} V_{d d}^{i j}$ with

$$
V_{d d}^{i j}=\frac{\mu_{0}}{4 \pi}\left[\frac{\vec{\mu}_{i} \cdot \vec{\mu}_{j}}{\left|\mathbf{r}_{i j}\right|^{3}}-\frac{3\left(\vec{\mu}_{i} \cdot \hat{\mathbf{r}}_{i j}\right)\left(\vec{\mu}_{j} \cdot \hat{\mathbf{r}}_{i j}\right)}{\left|\mathbf{r}_{i j}\right|^{3}}\right],
$$

where $\mu_{0}$ is the vacuum permeability, $\mathbf{r}_{i j}=\mathbf{r}_{i}-\mathbf{r}_{j}$, $\hat{\mathbf{r}}_{i j}=\mathbf{r}_{i j} /\left|\mathbf{r}_{i j}\right|, \mathbf{r}_{i}$ is the coordinate of the $i$-th site, and $\vec{\mu}_{i}=\gamma \mathbf{S}_{i}$ is the magnetic dipole moment at site $i$, with $\mathbf{S}_{i}=\hat{a}_{\alpha}^{\dagger}(i) \mathbf{F}_{\alpha \beta} \hat{a}_{\beta}(i)$ being the total angular momentum operator and $\gamma=g_{F} \mu_{B}$ the gyromagnetic ratio. Finally, the coupling of the atoms to the external magnetic field $\mathbf{B}_{\text {ext }}$ is described by

$$
H_{B}=-\gamma \sum_{i} \mathbf{S}_{i} \cdot \mathbf{B}_{\text {ext }}
$$

In this letter we consider a one-dimensional optical lattice along the $z$-direction, which we also choose as the quantization axis. Hence

$$
V_{d d}^{i j}=\frac{\mu_{0}}{4 \pi\left|\mathbf{r}_{i j}\right|^{3}}\left[\vec{\mu}_{i} \cdot \vec{\mu}_{j}-3 \mu_{i}^{z} \mu_{j}^{z}\right],
$$

with $\mu_{j}^{z}$ being the $z$-component of $\vec{\mu}_{j}$. In the absence of long-range magnetic dipole-dipole interaction and of external magnetic fields, the individual condensates can therefore be considered as independent "magnets" whose pseudo-spin vectors point in random directions, with no spin correlations between sites. Our goal is to determine the spin structure of the system if the different sites are allowed to interact with each other through the magnetic dipole-dipole interaction.

In the absence of spatial overlap between individual condensates, and neglecting unimportant constants, the total Hamiltonian of the system takes the form [12]

$$
\begin{aligned}
H= & \sum_{i}\left[\lambda_{a}^{\prime} \mathbf{S}_{i}^{2}+\gamma \sum_{j \neq i} \lambda_{i j} \mathbf{S}_{i} \cdot \mathbf{S}_{j}\right. \\
& \left.-3 \gamma \sum_{j \neq i} \lambda_{i j} S_{i}^{z} S_{j}^{z}-\gamma \mathbf{S}_{i} \cdot \mathbf{B}_{\mathrm{ext}}\right]
\end{aligned}
$$

where $\quad \lambda_{a}^{\prime}=(1 / 2) \lambda_{a} \int d^{3} r\left|\phi_{i}(\mathbf{r})\right|^{4}$ and $\lambda_{i j}=$ $\gamma \mu_{0} /\left(4 \pi\left|\mathbf{r}_{i j}\right|^{3}\right)$.

In general the external magnetic field consists of two contributions: a controlled, external applied field; and an effective "stray" field that accounts for all possible effects from the experimental environment and the system itself. A typical example is the environmental magnetic fluctuations. In the present case, we take the applied field along the quantization axis $z$. The effective environmental magnetic field, in contrast, can have any orientation. We choose it without loss of generality to be along the transverse direction, including any longitudinal component in the definition of the applied field. Hence the external field has the form

$$
\mathbf{B}_{\mathrm{ext}}=B_{z} \hat{\mathbf{z}}+B_{\rho} \hat{\rho},
$$

where $\rho=\sqrt{x^{2}+y^{2}}$ is the radial coordinate.

If the optical lattice is sufficiently long, one can safely neglect the boundary effects and concentrate on a generic single site $i$ of spin $\mathbf{S}$. Its Hamiltonian reads

$$
\begin{aligned}
h= & \lambda_{a}^{\prime} \mathbf{S}^{2}-\gamma \mathbf{S} \cdot\left[\left(B_{z}+2 \sum_{j \neq i} \lambda_{i j} S_{j}^{z}\right) \hat{\mathbf{z}}\right. \\
& \left.+\left(B_{\rho}-\sum_{j \neq i} \lambda_{i j} S_{j}^{\rho}\right) \hat{\rho}\right] .
\end{aligned}
$$

We determine its ground state in the mean-field approximation (also known as the Weiss molecular potential approximation) 13. It consists in replacing the operators $S_{j}^{\alpha}, \alpha=\rho, z$, by their ground-state expectation value

$$
\left\langle S_{j}^{\alpha}\right\rangle \rightarrow M_{\alpha}=N m_{\alpha}
$$

which is assumed to be the same for different sites. We remark that $m_{z}$ is nothing but the difference in population of the Zeeman sublevels of magnetic quantum numbers \pm 1 . This allows us to approximate the Hamiltonian (4) by

$$
h_{\mathrm{mf}}=\lambda_{a}^{\prime} \mathbf{S}^{2}-\gamma \mathbf{S} \cdot \mathbf{B}_{\mathrm{eff}}
$$

where we have introduced the effective magnetic field

$$
\mathbf{B}_{\mathrm{eff}}=\left(B_{z}+2 \Lambda m_{z}\right) \hat{\mathbf{z}}+\left(B_{x}-\Lambda m_{\rho}\right) \hat{\rho}
$$

and $\Lambda=N \sum_{j \neq i} \lambda_{i j}$.

We mentioned that the individual spinor condensates at the lattice sites are ferromagnetic, $\lambda_{a}^{\prime}<0$. In that case, the ground state of the mean-field Hamiltonian (6) must correspond to a situation where the condensate at the site $i$ under consideration must be aligned along $\mathbf{B}_{\text {eff }}$ and takes its maximum possible value $N$. That is, the ground state of the mean-field Hamiltonian (6) is simply

$$
|G S\rangle=|N, N\rangle_{\mathbf{B}_{\text {eff }}}
$$


where the first number denotes the total angular momentum and the second its component along the direction of $\mathbf{B}_{\text {eff }}$. Note that $|G S\rangle$ represents a spin coherent state in the basis of $\left|S, S_{z}\right\rangle$. The fact that the ground state magnetic dipole moment of each lattice site is $N$ times that of an individual atom results in a significant magnetic dipole-dipole interaction even for lattice points separated by hundreds of nanometers. This feature, which can be interpreted as a signature of Bose enhancement, is in stark contrast with usual ferromagnetism, where the magnetic interaction is negligible compared to exchange and where the use of fermions is essential [13].

The mean-field ground state of Eq. (8) allows us to calculate the magnetization components $m_{z}$ and $m_{x}$. One finds readily

$$
m_{\alpha}=\frac{1}{N}\left\langle G S\left|S_{i}^{\alpha}\right| G S\right\rangle=\cos \theta_{\alpha},
$$

where $\theta_{\alpha}$ is the angle between $\mathbf{B}_{\text {eff }}$ and the $\alpha$-axis.

For $B_{z}=0$, Eq. (9) yields

$$
\begin{aligned}
& m_{z}=\frac{2 \Lambda m_{z}}{\sqrt{\left(2 \Lambda m_{z}\right)^{2}+\left(B_{\rho}-\Lambda m_{\rho}\right)^{2}}}, \\
& m_{\rho}=\frac{B_{\rho}-\Lambda m_{\rho}}{\sqrt{\left(2 \Lambda m_{z}\right)^{2}+\left(B_{\rho}-\Lambda m_{\rho}\right)^{2}}} .
\end{aligned}
$$

The solutions to Eqs. (10) can be divided into two cases:

1. For $B_{\rho} \geq 3 \Lambda$, the only solutions are $m_{z}=0$ and $m_{\rho}=1$. That is, the lattice of condensates is magnetically polarized along the transverse magnetic field.

2. For $B_{\rho}<3 \Lambda$, there are two coexisting sets of solutions: (i) $m_{z}=0$ and $m_{\rho}=1$; and (ii) $m_{z}= \pm \sqrt{1-\left(B_{\rho} / 3 \Lambda\right)^{2}}$ and $m_{\rho}=B_{\rho} / 3 \Lambda$. However, it is easily seen that the state associated with the latter solutions has the lower energy. Hence it corresponds to the true ground state, while solution i) represents an unstable equilibrium.

We have, then, the following situation: As the environmental effective magnetic field strength is reduced below a critical value $3 \Lambda$, the lattice ceases to be polarized along the direction of that field. A phase transition occurs, and a spontaneous magnetization along the $z$-direction appears, characterized by a finite $m_{z}$.

This phenomenon is reminiscent of conventional ferromagnetism. Indeed, our model is somewhat analogous to the Ising model [1], with the environmental transverse magnetic field $B_{\rho}$ playing the role of temperature. For $B_{\rho}=0$ - corresponding to zero temperature in Ising model — the spins at each lattice site $\mathbf{S}_{i}$ align themselves along the lattice direction, even in the absence of longitudinal field. This spontaneous spin magnetization diminishes as $B_{\rho}$ increases, and completely vanishes if $B_{\rho}$ exceeds the critical value $3 \Lambda$ - the analog of the Curie temperature in the Ising model. We note however that the two cases exhibit important qualitative differences: For example, no spontaneous magnetization occurs in 1D Ising model, for any finite temperature [1].

Our analysis so far assumes an infinite one-dimensional lattice. This assumption is required for the validity of mean-field approximation. We note however that the appearance of a spontaneous magnetization does not rely on this condition being fulfilled. We demonstrate this point by considering just two lattice sites, that is, a doublewell situation. In this case, we can numerically solve the Hamiltonian (3), without invoking the mean-field approximation, by expanding the Hamiltonian matrix on the basis of $\left|S_{1}=N, S_{1}^{z}\right\rangle \otimes\left|S_{2}=N, S_{2}^{z}\right\rangle$. Figure 1 1 shows the spontaneous magnetization $m_{z}$ as a function of the external field strength $B_{\rho}$. As the Hamiltonian matrix has a size of $(2 N+1)^{2} \times(2 N+1)^{2}$, we cannot use a very large $N$. However, as we can see from the figure, as $N$ increases, $m_{z}$ rapidly approaches the mean-field results. Hence the mean-field treatment is in fact a surprisingly good approximation for our purpose.

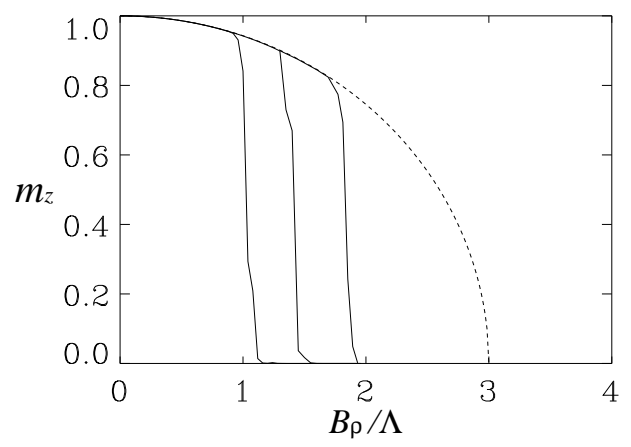

FIG. 1. Spontaneous magnetization as a function of environmental magnetic field strength. Only the positive values are plotted. The dashed line represents the mean-field result $m_{z}=\sqrt{1-\left(B_{x} / 3 \Lambda\right)^{2}}$ and the solid lines, from left to right, correspond to the exact numerical results for a two-site lattice with $N=10,15$ and 25 atoms, respectively.

To estimate the feasibility of an experimental detection of the spontaneous magnetization, we consider as an example the $F=1$ electronic ground state $\left({ }^{3} \mathrm{~S}_{1 / 2}\right)$ of ${ }^{87} \mathrm{Rb}$, for which $\lambda_{a}^{\prime}<0$ [2]:3]. The Landé factor for this state is $g_{F}=-1 / 2$. For an optical potential of period equal to $426 \mathrm{~nm}$ (nearest neighbor separation) we find $\Lambda=N \sum_{j \neq i} \lambda_{i j} \approx 1.6 N \times 10^{-7} \mathrm{G}$. If the particle number at each site is $N=2000$, this gives a critical value of the environmental magnetic field of $3 \Lambda=1 \mathrm{mG}$. These numbers indicate that the observation of spontaneous magnetization in a lattice of spinor condensates is well within experimental reach.

In a typical experimental situation, the environmental magnetic field is likely to exhibit temporal fluctuations. To account for them, we assume that this field has a uniform angular distribution and that the fluctuations in field strength have a width $\Delta_{B}$. The time-averaged environmental field is again assumed to be along the trans- 
verse direction, with strength $B_{\rho}$. Assuming the magnetization of the system follows the field fluctuations adiabatically, it is not difficult to include this effect into our mean-field treatment. The time-averaged spontaneous magnetization along $z$-axis under satisfies then the cubic equation

$$
\left(1-m_{z}^{2}\right)\left(9 \Lambda^{2} m_{z}^{2}+\Delta_{B}^{2} / 2\right)=\left(B_{\rho}^{2}+\Delta_{B}^{2}\right) m_{z}^{2} .
$$

Figure 2 illustrates $m_{z}$ as a function of $B_{\rho}$ for various $\Delta_{B}$. As we can see, the effect of the field fluctuation is to decrease the maximum spontaneous magnetization, and to increase the critical field strength at which the spontaneous magnetization vanishes. The maximun $m_{z}$ at $B_{\rho}=0$ decreases from 1 to $1 / 3$ when $\Delta_{B}$ increases from 0 to infinity.

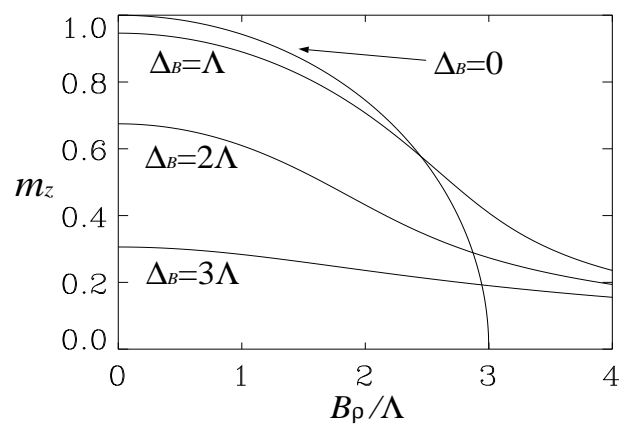

FIG. 2. Mean-field results of the time-averaged spontaneous magnetization as a function of environmental magnetic field strength, taking the magnetic field fluctuations into accout. Only the positive values are plotted.

Recently, condensates trapped in periodic optical potentials have attracted much attention. The phase coherence and atom statistics of the system have been studied experimentally [4, 14, 15]. However their magnetic properties have not been fully explored. A few years ago, Meacher et al. observed experimentally the paramagnetic behavior of cold (but non-condensed) cesium atoms confined in an optical lattice [16]. Here we have demonstrated that by replacing the cold atoms with a spinor condensate, ferromagnetism can be observed. This is made possible by the collectively enhanced magnetic moments of the condensate which in turn enhances magnetic dipole-dipole interaction between different lattice sites.

In future work it will be interesting to study the dynamical response of the system under the effect of an external time-dependent longitudinal magnetic field. The instantaneous magnetization in this case is likely to form hysteresis loops which might find applications e.g. in quantum information processing. Further studies should also include the properties of finite temperature excitations of the system, which correspond to spin waves. The extension of this work beyond one-dimensional lattices also shows much promise: Although it is predominantly ferromagnetic in $1 \mathrm{D}$, the dipole-dipole interaction is anisotropic and contains both ferromagnetic and antiferromagnetic terms. In higher-dimensional lattices, the ground-state spin structure will thus become much richer.

We conclude by remarking that in addition to this intrinsic interest, the study of the magnetic properties of spinor condensate lattices provide us with a highly controllable test system to study fundamental static and dynamical aspects of magnetism and lattice dynamics, including, e.g., the role of dimensionality in phase transitions and macroscopic quantum tunneling of the magnetic moments.

This work is supported in part by the US Office of Naval Research under Contract No. 14-91-J1205, by the National Science Foundation under Grant No. PHY9801099, by the US Army Research Office, by NASA, and by the Joint Services Optics Program.

[1] See for example, Kerson Huang, Statistical Mechanics (John Wiley \& Sons, New York, 1987).

[2] D. Heinzen, private communication (2001).

[3] J. P. Burke, Jr. and J. L. Bohn, Phys. Rev. A 59, 1303 (1999).

[4] B. P. Anderson and M. A. Kasevich, Science 281, 1686 (1998).

[5] D. Jaksch et al., Phys. Rev. Lett. 81, 3108 (1998).

[6] M. Raizen, private communication (2001)

[7] R. Carretero-Gonzalez and K. Promislow, e-print condmat/0105600, (2001).

[8] J. Stenger et al., Nature (London) 396, 345 (1998).

[9] W. Zhang and D. F. Walls, Phys. Rev. A 57, 1248 (1998).

[10] T. -L. Ho, Phys. Rev. Lett. 81, 742 (1998).

[11] T. Ohmi and K. Machida, J. Phys. Soc. Jpn. 67, 1882 (1998).

[12] C. K. Law, H. Pu and N. P. Bigelow, Phys. Rev. Lett. 81, 5257 (1998).

[13] N. W. Ashcroft and N. D. Mermin, Solid State Physics (Harcourt Brace College Publishers, New York, 1976).

[14] C. Orzel et al., Science, 291, 2386 (2001).

[15] M. Greiner et al., e-preprint cond-mat/0105105.

[16] D. R. Meacher et al., Phys. Rev. Lett. 74, 1958 (1995). 